\title{
Identification of a Dutch founder mutation in MUSK causing fetal akinesia deformation sequence
}

\author{
M Brigita Tan-Sindhunata ${ }^{1}$, Inge B Mathijssen ${ }^{2}$, Margriet Smit ${ }^{1}$, Frank Baas ${ }^{3}$, Johanna I de Vries ${ }^{4}$, \\ J Patrick van der Voorn ${ }^{5}$, Irma Kluijt ${ }^{2}$, Marleen A Hagen ${ }^{4}$, Eveline W Blom ${ }^{6}$, Erik Sistermans ${ }^{1}$, \\ Hanne Meijers-Heijboer ${ }^{1,2}$, Quinten Waisfisz ${ }^{1}$, Marjan M Weiss ${ }^{1,8}$ and Alexander J Groffen ${ }^{\star 1,7,8}$
}

Fetal akinesia deformation sequence (FADS) refers to a clinically and genetically heterogeneous group of disorders with congenital malformations related to impaired fetal movement. FADS can result from mutations in CHRNG, CHRNA1, CHRND, DOK7 and RAPSN; however, these genes only account for a minority of cases. Here we identify MUSK as a novel cause of lethal FADS. Fourteen affected fetuses from a Dutch genetic isolate were traced back to common ancestors 11 generations ago. Homozygosity mapping in two fetuses revealed MUSK as a candidate gene. All tested cases carried an identical homozygous variant $\mathrm{C}$.1724T $>$ C; p.(Ile575Thr) in the intracellular domain of MUSK. The carrier frequency in the genetic isolate was $8 \%$, exclusively found in heterozygous carriers. Consistent with the established role of MUSK as a tyrosine kinase that orchestrates neuromuscular synaptogenesis, the fetal myopathy was accompanied by impaired acetylcholine receptor clustering and reduced tyrosine kinase activity at motor nerve endings. A functional assay in myocytes derived from human fetuses confirmed that the variant blocks MUSK-dependent motor endplate formation. Taken together, the results strongly support a causal role of this founder mutation in MUSK, further expanding the gene set associated with FADS and offering new opportunities for prenatal genetic testing. European Journal of Human Genetics (2015) 23, 1151-1157; doi:10.1038/ejhg.2014.273; published online 24 December 2014

\section{INTRODUCTION}

Fetal akinesia deformation sequence (FADS; MIM 208150) represents a group of disorders characterized by decreased or absent fetal movements. Affected infants may die in utero or shortly after birth due to severe pulmonary hypoplasia. FADS was first recognized in 1974 by Pena and Shokeir, and therefore was originally called the Pena-Shokeir syndrome. ${ }^{1,2}$ The etiology of FADS is heterogeneous, including neuromuscular disorders, metabolic diseases, syndromic and environmental causes. ${ }^{3}$ Mutations in genes expressed at the neuromuscular junction (NMJ) are increasingly recognized as important causes of FADS, including CHRNA1 (MIM 100690), CHRND (MIM 100720), CHRNG (MIM 100730), DOK7 (MIM 610285) and RAPSN (MIM 601592). ${ }^{3}$ These genes however explain a minority of cases: in a cohort of 59 kindreds with Lethal Multiple Pterygium Syndrome (LMPS [MIM 253290]) and FADS, it was estimated that 24\% were positive for mutations in either of these genes. ${ }^{4}$

Here we describe a novel founder mutation in MUSK, encoding a muscle-specific tyrosine kinase essential for NMJ development ${ }^{5}$ as a frequent cause of lethal FADS in a Dutch genetic isolate. We demonstrate that this variant causes a defect in neuromuscular synaptogenesis.

\section{MATERIALS AND METHODS}

Informed consent was given by all subjects included in this study. For homozygosity mapping in two fetuses affected with FADS (case 10 and
11 in Table 1), an Affymetrix 6.0 array was used. Overlapping regions of homozygosity were analyzed for candidate genes ('Genomic Oligoarray and SNP array evaluation tool v1.0 $\left.0^{5}\right){ }^{6}$ Sanger sequencing of the coding region and flanking intronic regions of MUSK (primer details available on request) was performed on a ABI 3730 type DNA analyzer (Applied Biosystems, Foster City, CA, USA). The variant was deposited in the Leiden Open Variation Database at www.lovd.nl (patient ID no. 0033104). After routine autopsy, various fetal muscle tissues ( $\mathrm{m}$. quadriceps, $\mathrm{m}$. psoas, $\mathrm{m}$. biceps femoris, diaphragm) were collected for vital and nonvital assays, including hematoxylin and eosin staining, elastic Van Gieson staining and ATPase assays to distinguish type I and type II muscle fibers. To visualize NMJs, cryosections were fixed with $4 \%$ paraformaldehyde and stained for synaptotagmin-1 (rabbit polyclonal W855, 1:500), phosphotyrosine (mouse monoclonal 4G10, 1:200, Millipore, AmsterdamZuidoost, Netherlands) ${ }^{7}$ and acetylcholine receptors ( $\alpha$-bungarotoxin-Rhodamine, 1:1000, Sigma, St Louis, MO, USA). For a side-by-side comparison of wild-type and mutant motor endplates of similar developmental age, stainings of case 8 (23 weeks of gestation) were performed in parallel with that of a control fetus aborted due to cervical insufficiency (22 weeks of gestation).

To assay MUSK-induced motor endplate maturation, fetal myocytes from a fetus homozygous for the c.1724T $>\mathrm{C}$; $\mathrm{p}$. (Ile575Thr) variant were maintained in DMEM supplemented with $20 \%$ fetal bovine serum, glutaMAX and penicillin/streptomycin at $37^{\circ} \mathrm{C}$ in $5 \% \mathrm{CO}_{2}$. Myotubes were induced by growing the myocytes in $2 \%$ serum on coverslips coated with $72 \mu \mathrm{g} / \mathrm{ml}$ Matrigel (BD Biosciences). After 1 day in vitro (DIV1), myocytes were transduced with lentiviral vectors. Wild-type or mutant MUSK was engineered to contain a Flag tag in the ectodomain, a strategy previously shown to preserve MUSK activity. ${ }^{8}$ For efficient and safe cellular delivery, a third-generation

\footnotetext{
${ }^{1}$ Department of Clinical Genetics, VU University Medical Center, Amsterdam, The Netherlands; ${ }^{2}$ Department of Clinical Genetics, Academic Medical Center, Amsterdam, The Netherlands; ${ }^{3}$ Department of Genome Analysis, Academic Medical Center, Amsterdam, The Netherlands; ${ }^{4}$ Department of Obstetrics and Gynaecology, Research Institute MOVE, VU University Medical Center, Amsterdam, The Netherlands; ${ }^{5}$ Department of Pathology, VU University Medical Center, Amsterdam, The Netherlands; ${ }^{6}$ Department of Clinical Genetics, MUMC, Maastricht, The Netherlands; ${ }^{7}$ Department of Functional Genomics, Center for Neurogenomics and Cognition Research, VU University, Amsterdam, The Netherlands

*Correspondence: Dr AJ Groffen, Department of Clinical Genetics, VU University Medical Center, De Boelelaan 1085, 1081 HV Amsterdam, The Netherlands. Tel: +31 20 5986928; Fax: +31 20 5986926; E-mail: a.j.a.groffen@vu.nl

8These authors contributed equally to this work.
}

Received 11 August 2014; revised 29 October 2014; accepted 10 November 2014; published online 24 December 2014 


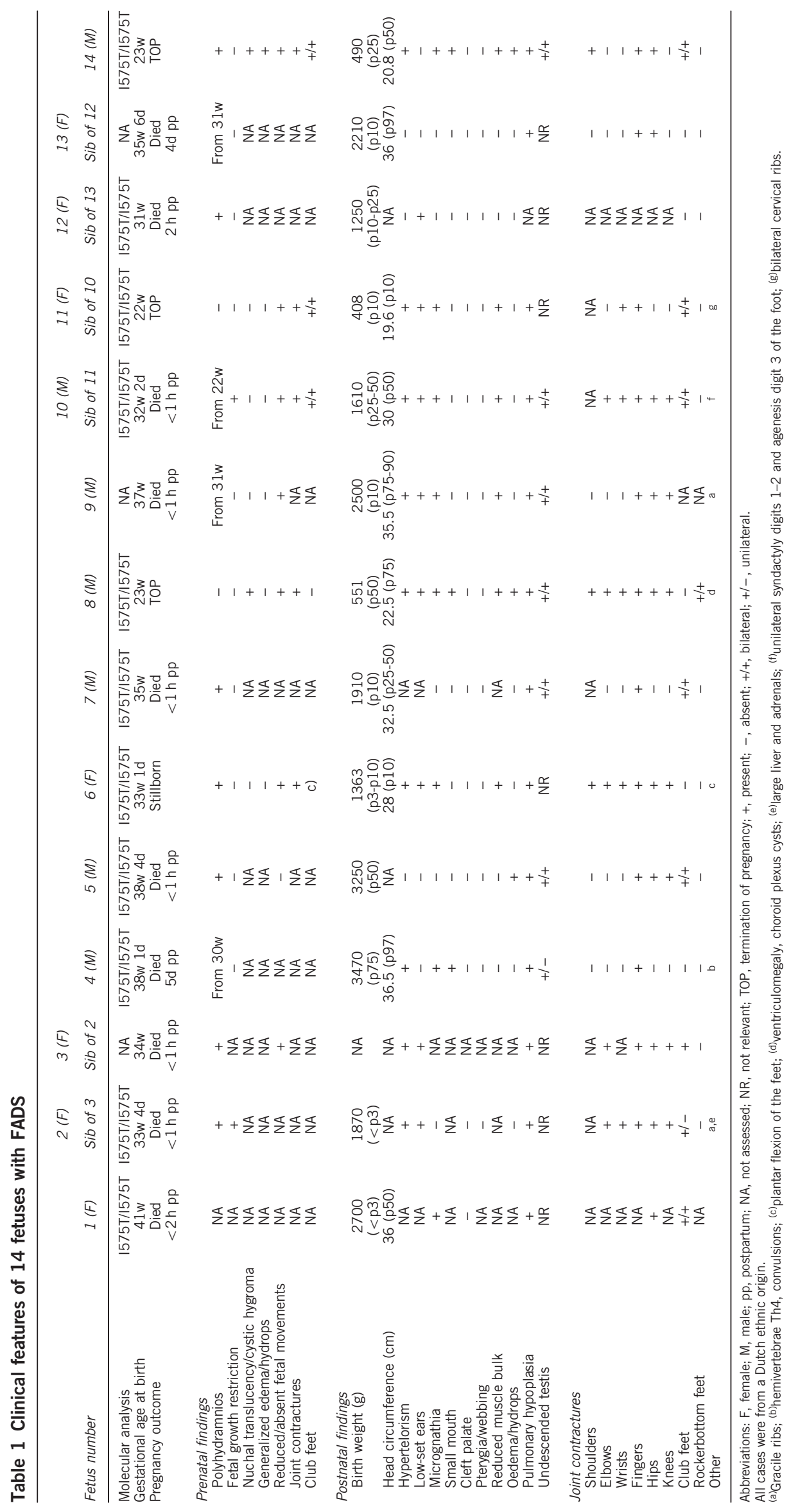


a

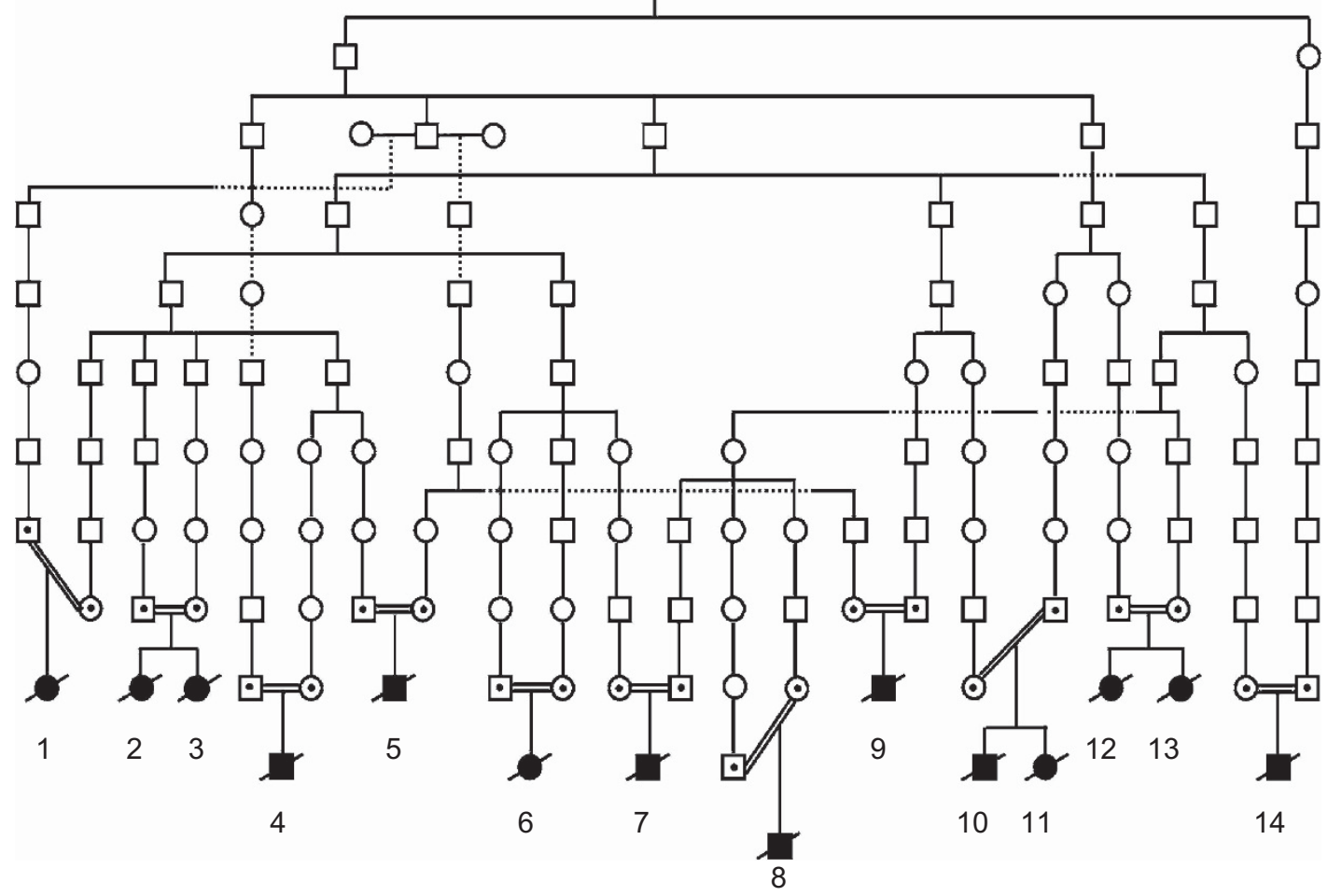

b

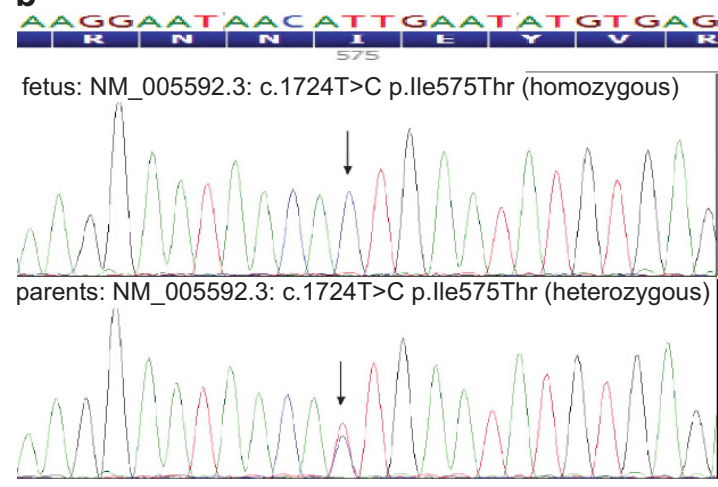

Ile575

C

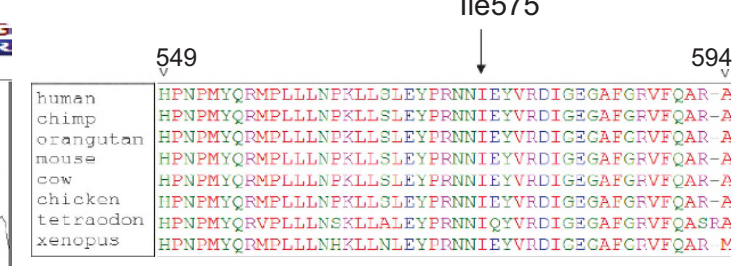

88

Extracellular

88

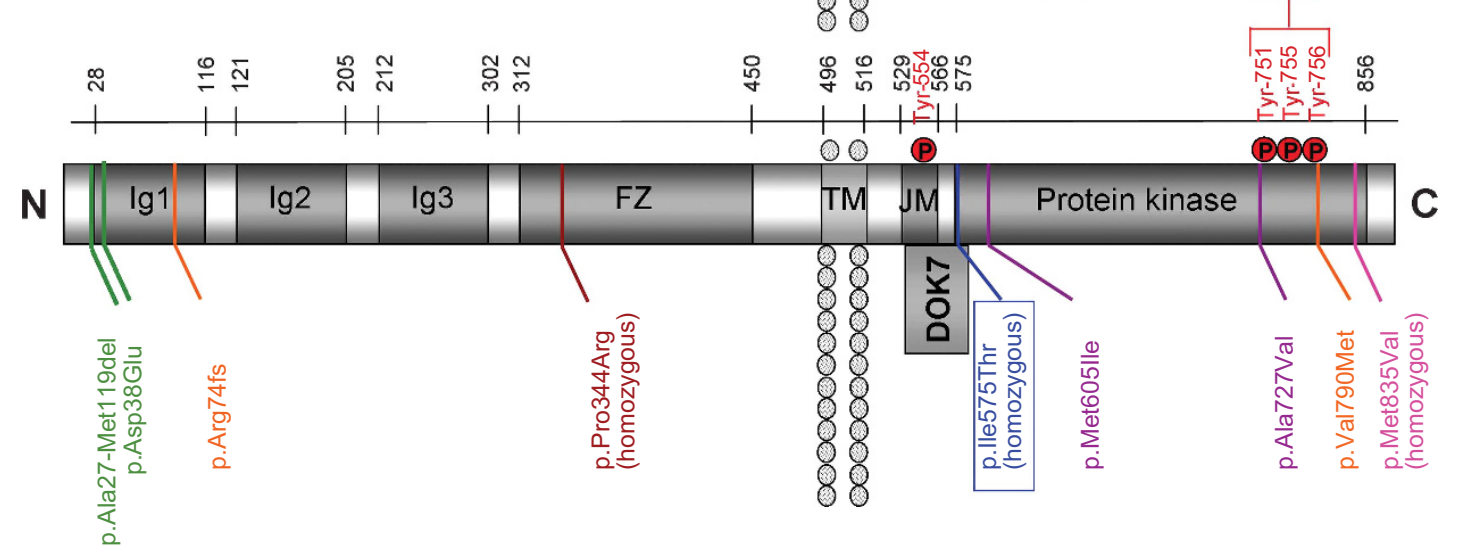

Figure 1 For caption please see next page. 
lentiviral expression system was used. ${ }^{9}$ At DIV6, acetylcholine receptor (AChR) cluster formation was induced by extracellular administration of $100 \mathrm{ng} / \mathrm{ml}$ neuronal agrin (splice variant 3,4,8; R\&D Systems, Minneapolis, MN, USA). ${ }^{10}$ At DIV8, the myocytes were fixed with $4 \%$ paraformaldehyde, washed in Trisbuffered saline containing $0.1 \%$ Tween-20 (TBST) and stained with DAPI (1:2000) and $\alpha$-bungarotoxin-Rhodamine $(1: 2000)$ in TBST for $48 \mathrm{~h}$ at $4{ }^{\circ} \mathrm{C}$. The cells were washed again in TBST and mounted in Mowiol, followed by image acquisition on a custom-build FAINT microscope (Chromaphor, Oberhausen, Germany). AChR clusters were counted in $n=35$ images from three coverslips per condition, and a Student's $t$-test was used to compare the mean cluster density.

\section{RESULTS}

Homozygosity mapping in two fetuses with FADS from a single consanguineous family (case 10 and 11 in Table 1 and Figure 1a) identified MUSK as the prime candidate gene. Sanger sequencing revealed a novel homozygous missense variant (NM_005592.3: c.1724T > C; p.(Ile575Thr); Figure 1b) absent in relevant databases (dbSNP build 132, 1000 Genomes project, and Exome Variant Server data ESP5400). The amino-acid change was predicted to be potentially pathogenic by various bioinformatics tools: SIFT: Deleterious (score: 0, median: 4.32); Align GVGD: C65 (GV: 0.00-GD: 89.28); PhyloP: 4.97 (highly conserved); Mutation Taster: disease causing, prob: 0.9999999987; Polyphen: probably damaging (HumVar score 0.999). The variant was also absent in a cohort of 1326 Dutch controls, analyzed by Taqman SNP Genotyping (Life Technologies).

As the affected fetuses originated from a genetic isolate, we hypothesized the occurrence of a founder effect. Twelve other cases from 10 families from the same genetic isolate came to our clinic between 1980 and 2012. DNA was available from nine cases. All carried the same homozygous variant and all parents were heterozygous carriers. All 14 cases had common ancestors traced back 11 generations (Figure 1a). Consistent with a founder effect, the MUSK variant co-segregated with a shared haplotype around the MUSK gene (Supplementary Table 1). Genetic testing of 530 healthy controls from the genetic isolate revealed no homozygous individuals and 43 heterozygous carriers, resulting in a carrier frequency of $8 \%$ in this region. Subsequently, a cohort of 26 unresolved LMPS/FADS cases who did not come from the genetic isolate were tested by Sanger sequencing, revealing no likely pathogenic variants in MUSK.

The clinical symptoms available from 14 affected fetuses are summarized in Table 1. Pictures of four fetuses are shown in Figure 2. The pregnancy was terminated in three fetuses, whereas the natural course of the pregnancy was followed in 11 fetuses. From the naturally born fetuses, seven were born prematurely between 31-37 weeks of gestation and four were born at term. All 11 were stillborn or died shortly after birth, usually after failed resuscitation. Typical prenatal ultrasound findings were polyhydramnios, progressively reduced fetal movements and joint contractures. The polyhydramnios generally developed in the second or third trimesters, and became severely pronounced around 30 weeks, sometimes leading to premature rupture of membranes or premature contractions. Postmortem examination revealed severe pulmonary hypoplasia in all cases. Hypertelorism, low-set ears, micrognathia and reduced muscle bulk were seen in most fetuses. The contractures could affect all joints with variable severity; however, all fetuses had contractures of the fingers (camptodactyly) and 10 out of 13 analyzed fetuses had severely affected feet (club feet, plantar flexion or rocker-bottom feet). Two fetuses who died 4 and 5 days after birth were severely hypotonic. All male fetuses had cryptorchidism, although this may not be representative because most children were born prematurely. Three of twelve analyzed fetuses were mildly edematous. Gracile ribs were observed in two cases. Some additional findings were observed only in

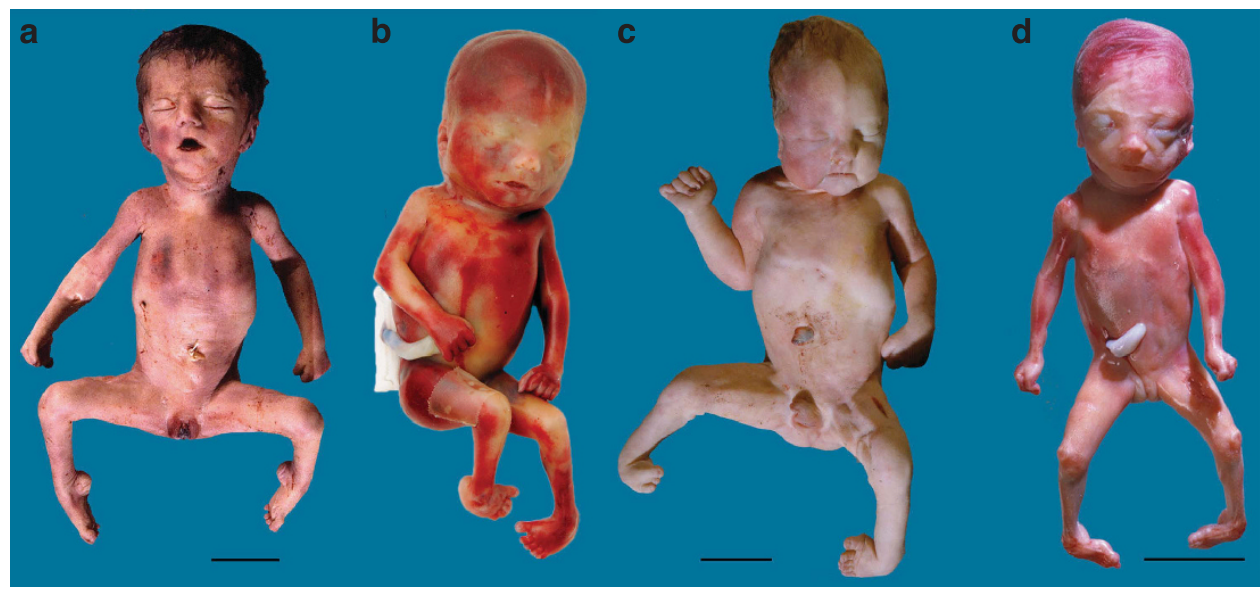

Figure 2 Macroscopic appearance of four fetuses homozygous for the c.1724T>C; p.(Ile575Thr) variant in MUSK. (a) Case 6, stillborn at a gestational age of 33 weeks; (b) case 8, termination of pregnancy at 23 weeks; (c) case 10, died immediately after birth at 32 weeks; (d) case 11, termination of pregnancy at 22 weeks. Note the multiple contractures, facial appearance of hypertelorism and micro-retrognathia, club feet (b-d), oligodactyly of the right foot (c), plantar flexion of the feet (a). For details see Table 1. Bars indicate $5 \mathrm{~cm}$.

Figure 1 Co-segregation of a variant in MUSK with lethal FADS in a Dutch genetic isolate. (a) Schematic illustrating the common ancestry of 14 affected fetuses from 11 families. (b) Sanger sequencing chromatograms of fetal (top) and parental (bottom) DNA samples identifying the variant NM_005592.3: c.1724T>C; p.(Ile575Thr) and multispecies alignment of the affected residue (right). (c) Domain structure of MUSK depicting the extracellular domain containing lgG-like domains, a Frizzled domain (FZ, also named C6 box), a transmembrane domain (TM) and a cytoplasmic domain that contains a juxtamembrane (JM)/PTB-domain and the tyrosine kinase domain. Ile575 is located at the boundary between the JM and kinase domains, very close to the Dok7-interacting region and the autophosphorylation site Tyr554. Other mutations previously reported to cause CMS are also indicated. Mutations found in compound heterozygotes are color-matched ${ }^{23-28}$. All amino-acid numbers refer to MUSK isoform 1 (GenPept accession NP_005583.1). 

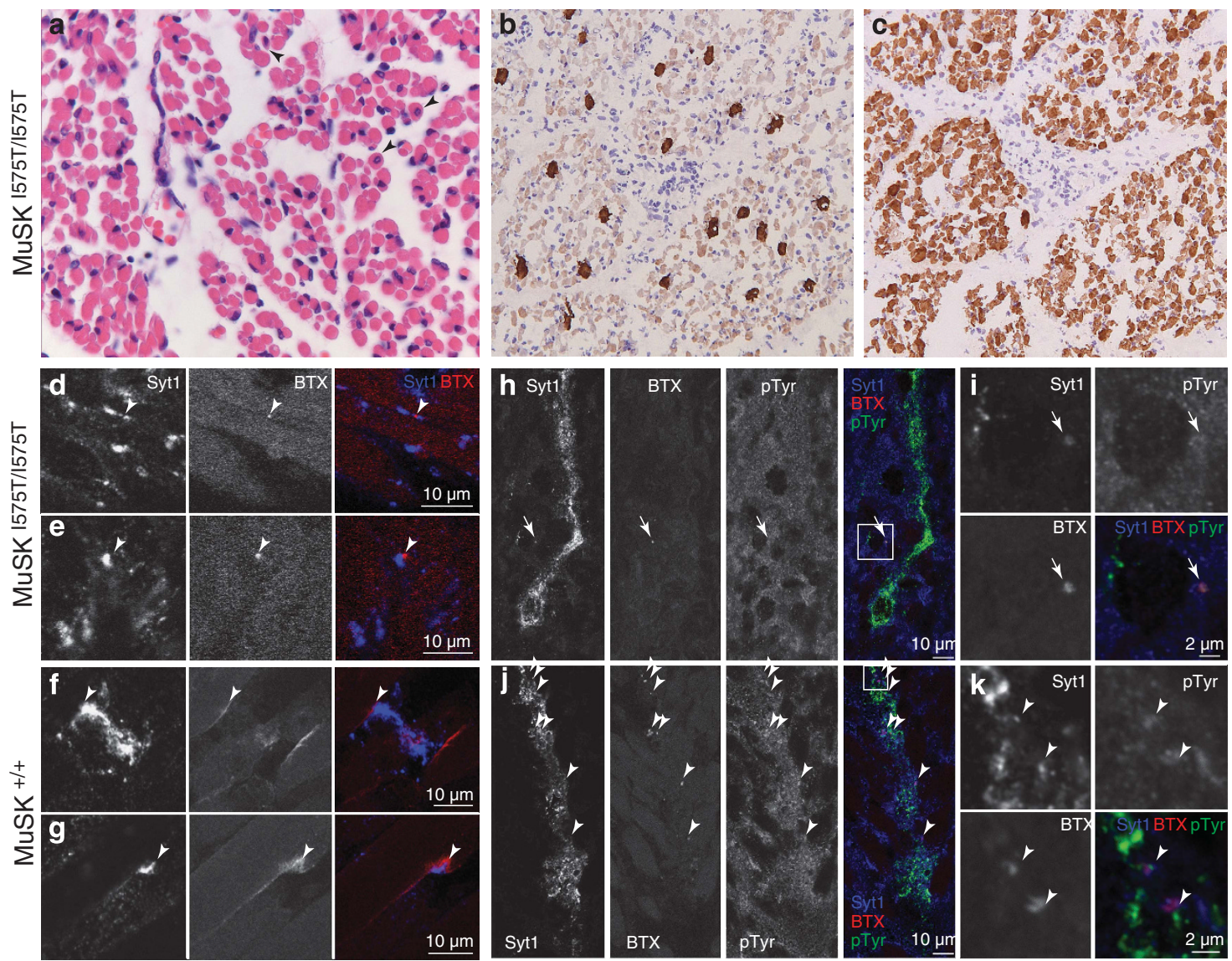

Figure 3 Histopathological findings indicative for defects in neuromuscular synaptogenesis. (a) Skeletal muscle biopsies showed small and rounded atrophic fibers and an increased number of intracellular nuclei (arrowheads). (b and c) Immunostaining of type I (b) and type II fibers (c) indicated a relative loss of type I fibers. (d-g) Combined staining for nerve terminals (synaptotagmin- 1 ) and AChR clusters ( $\alpha$-bungarotoxin-rhodamine) in quadriceps muscle indicated fewer and smaller neuromuscular junctions in case 8 (arrowheads in $\mathbf{d}, \mathbf{e} ; 23$ weeks of gestation) compared with a control fetus (f, $\mathbf{g}$; 22 weeks). (h-k) Combined staining for nerve terminals, AChR clusters and anti-phosphotyrosine (pTyr) monoclonal $4 \mathrm{G} 10$ in diaphragm indicated fewer clusters (arrowheads) and a loss of tyrosine kinase activity in MUSK $K^{1575 T / 1575 T}$ endplates $(\mathbf{h}, \mathbf{i})$ compared with control tissue (j, k). Note that residual tyrosine kinase activity was detected in some mutant endplates (arrow), which may also result from unrelated tyrosine kinases.

a single fetus and are considered to be incidental: cervical ribs, mild ventriculomegaly, choroid plexus cysts, a solitary hemivertebra, syndactyly of the first and second toes and agenesis of the third toe. None of the fetuses had pterygia or a cleft palate.

Microscopic examination of tissues from case 8 (a terminated pregnancy at 23 weeks of gestation) showed no abnormalities of the cerebrum, cerebellum, cranial nerve motor nuclei and spinal motor neurons. In contrast, muscle tissues (m. quadriceps, m. psoas, $\mathrm{m}$. biceps femoris, diaphragm) showed several myopathic features consistent with a denervating or neurogenic cause (Figure 3). There was clear variation in muscle fiber diameter with small and rounded shaped atrophic fibers and there was a mild increase in endomysial fibrosis. The number of internal nuclei was increased consistent with maturational delay. The ratio between type 1 and 2 fibers was 1:10 suggesting a loss of type 1 (slow) fibers (Figure $3 \mathrm{~b}$ and $\mathrm{c}$ ).

MUSK is a receptor tyrosine kinase expressed on the muscle cell membrane. ${ }^{5,11}$ It consists of an extracellular domain containing IgG-like domains, a Frizzled-like cysteine-rich domain (C6 box), a transmembrane domain and a cytoplasmic domain composed of a juxtamembrane/PTB domain and a tyrosine kinase domain (Figure 1c). ${ }^{12,13}$ The variant c.1724T $>$ C; p. (Ile575Thr) is located in a highly conserved region at the boundary of the juxtamembrane and kinase domains. The important role of both domains in tyrosine kinase activation and Dok-7 signaling suggests that the variant may affect MUSK signaling and AChR clustering. ${ }^{14,15}$ To investigate this, local tyrosine kinase activity and AChR clusters were visualized at nascent motor endplates in muscle biopsies from an affected fetus (case 8, 23 weeks of gestation) and a control fetus aborted at 22 weeks due to cervical insufficiency (Figure $3 \mathrm{~d}-\mathrm{k}$ ). Ingrowing axons from motor neurons were visualized with the synaptic vesicle marker Synaptotagmin-1. In wild-type tissue, developing motor endplates were closely associated with AChR clusters, stained by $\alpha$-bungarotoxin-Rhodamine. In MUSK mutant muscle, these clusters were strongly reduced in number and appeared very small (Figure $3 \mathrm{~d}$, e and $\mathrm{h}$ ). Whereas motor endplates in the control fetus showed increased tyrosine kinase activity (Figure $3 j$ and $k$ ), the small AChR clusters in the FADS patient did not accumulate phosphotyrosines (Figure $3 \mathrm{~h}$ and i). Thus, histological examination was consistent with a defect in the MUSK signaling pathway.

To corroborate the above findings in fetal muscle tissue, we next investigated the AChR-clustering activity of mutant and wild-type MUSK in a direct comparison. Wild-type and mutant MUSK were expressed in HEK293 cells with similar molecular mass (Figure 4c), migrating in SDS-PAGE as a $110-\mathrm{kDa}$ protein consistent with previous studies. ${ }^{16,17}$ In myocytes derived from a fetus homozygous for the c.1724T > C variant, overexpression of wild-type MUSK significantly 


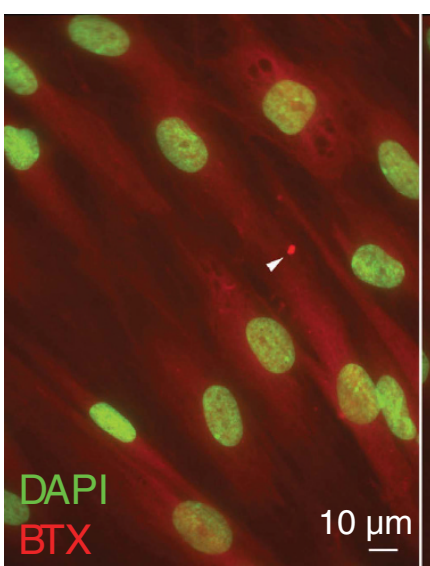

b

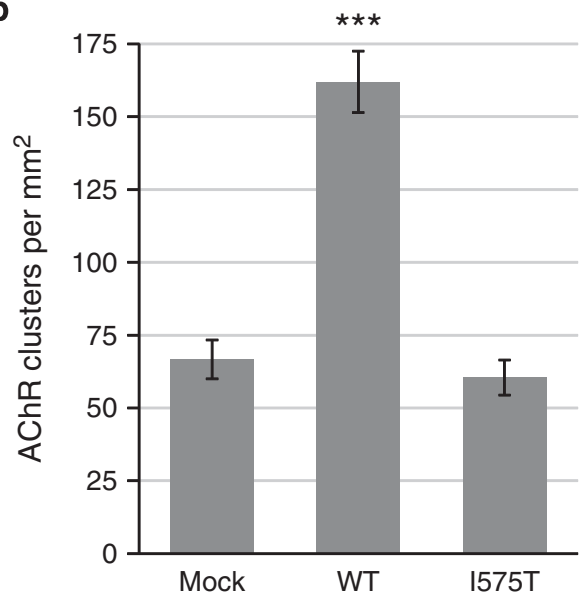

WT

I575T
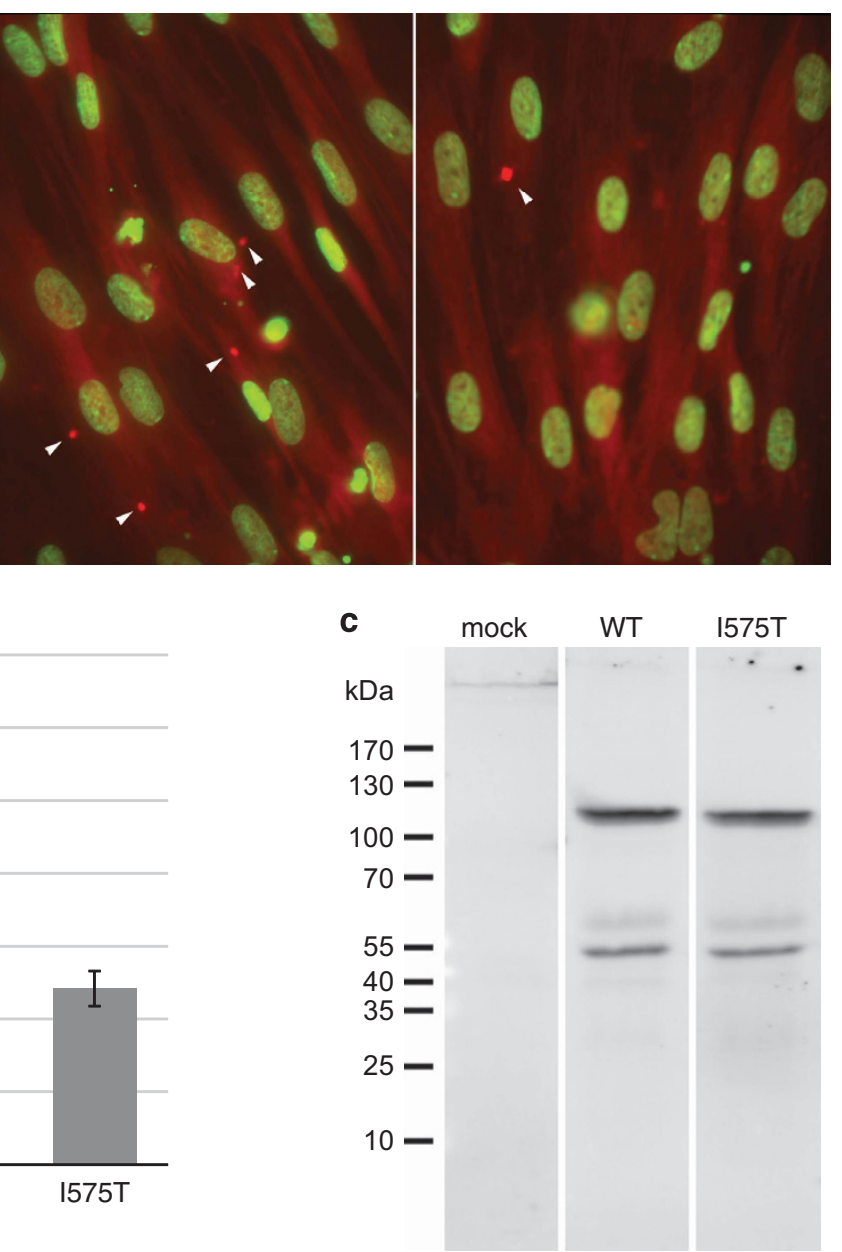

Figure 4 Wild-type but not mutant MUSK augments AChR clustering in MUSK ${ }^{1575 T}$ myocytes. (a) Primary myocytes derived from an affected fetus homozygous for MUSK ${ }^{1575 T}$ were plated on Matrigel-coated coverslips and transduced with lentiviral vectors encoding either MUSKWT or MUSK ${ }^{1575 T}$. As a control, non-transduced MUSK ${ }^{1575 T}$ cells were used. Myocytes were treated with neuronal Agrin $(3,4,8)$ and stained for nuclei (DAPI, displayed in green) and AChR clusters (Rhodamine-bungarotoxin, red). Representative examples from each experimental group are shown. (b) Mean \pm SEM values for the AChR cluster density, quantitated from 35 images from three coverslips of each group. ${ }^{* * *} P<0.001$ (Student's $t$-test). (c) Immunoblot analysis of Flag-tagged MUSK ${ }^{W T}$ and MUSK ${ }^{1575 T}$ constructs expressed in HEK293 cell lysates, suggesting similar yield and molecular mass.

increased the density of agrin-induced AChR clusters $(P<0.001$, $n=35)$, whereas mutant MUSK $^{\mathrm{I} 75 \mathrm{~T}}$ did not $(P=0.65, n=35$; see Figure $4 \mathrm{a}$ and $\mathrm{b})$. Taken together, our observations demonstrate that the c.1724T $>$ C; p.(Ile575Thr) variant strongly affects MUSK autoactivation, tyrosine phosphorylation and AChR clustering in both fetal tissue and cultured myocytes.

\section{DISCUSSION}

Mutations in MUSK, RAPSN, CHRNA1, CHRNB1, CHRND, CHRNE, CHRNG and DOK7 can also cause Congenital Myasthenic Syndromes (CMSs; MIM 608931, 608930, 601462). ${ }^{18}$ The severity of the mutation likely determines the severity of the phenotype ranging from CMS to lethal FADS. ${ }^{19-22}$ For CHRNG, a recent study has shown intra- and interfamilial variability for the same mutation and the severity of the phenotype (LMPS/FADS versus the nonlethal Escobar Variant of Multiple Pterygium Syndrome; MIM 265000), suggesting that there may be genetic and/or environmental modifiers. ${ }^{4}$

Previously, recessive mutations in MUSK were found in 11 individuals from five families with CMS. Affected subjects in the first family were compound heterozygous for a frameshift (p.Arg74fs) and missense mutation (p.Val790Met). ${ }^{23}$ In the second consanguineous family a homozygous missense mutation was found (p.Pro344Arg in the cysteine-rich extracellular domain). ${ }^{24}$ The third family included an individual with heteroallelic missense mutations (p.Met605Ile and p.Ala727Val) both located in the kinase domain. ${ }^{25}$ An Iranian case from consanguineous parents was homozygous for a missense mutation (p.Met835Val). ${ }^{26}$ Finally, two brothers from Turkish origin with a salbutamol-responsive limb-girdle CMS were compound heterozygous for a missense mutation (p.Asp38Glu) and a genomic deletion of exons 2-3. ${ }^{27}$ The clinical symptoms of these 11 CMS cases consisted of a variable, fatigable, predominantly proximal muscle weakness, partial ophthalmoparesis, mild ptosis and facial weakness. None of the patients had congenital joint contractures. Although there could be severe respiratory failure and hypotonia in the neonatal period, the condition seemed to improve during childhood and there was no delay of initial motor milestones.

Comparing the clinical and histological findings, the c.1724T >C; p.(Ile575Thr) variant appears to halt neuromuscular synaptogenesis at 
an early stage, and cause a more severe loss of function than the previously observed mutations, which cause the milder CMS phenotype. The relatively severe effect of the mutation may be explained by its location very close to the Dok7 interaction site, critically involved in MUSK dimerization and its tyrosine kinase activation. ${ }^{15}$ Consistent with this hypothesis, the clinical phenotype of the MUSK ${ }^{1575 T / 1575 T}$ fetuses is reminiscent of that of Musk knockout mice that fail to form stable NMJs, resulting in paralyzed embryos that die perinatally before taking a single breath. ${ }^{5}$

In conclusion, the novel founder mutation in MUSK is a major cause of lethal FADS in the studied genetic isolate and caused early defects in motor endplate development including decreased tyrosine kinase activity and a lack of AChR cluster formation. Although the absence of pterygia is noteworthy, this feature is shared with RAPSNand DOK7-associated FADS phenotypes. ${ }^{4}$ As clinical heterogeneity further obscures genotype-phenotype correlations, a molecular genetic analysis of the gene set involved in FADS/LMPS is probably the most effective classification method for this disease spectrum. The addition of MUSK to this gene set strengthens the existing idea that genes encoding neuromuscular synaptogenesis can cause phenotypes with variable severity, ranging from late-onset and neonatal CMS to the peri- or prenatal lethality observed in fetal akinesia.

\section{CONFLICT OF INTEREST}

The authors declare no conflict of interest.

\section{ACKNOWLEDGEMENTS}

We are grateful to the families for their cooperation. We thank Bertus Kuyt and Loes Stoets for the genealogy data, Alessandra Maugeri for helping with the Sanger sequencing analysis and Ruud Wolterman for tissue culture of primary myocytes. This work was financially supported by the Netherlands Genomics Initiative at the Centre for Medical Systems Biology (CMSB2) and the Netherlands Organization for Health Research and Development (ZonMW, project 91113022)

1 Hall JG: Pena-Shokeir phenotype (fetal akinesia deformation sequence) revisited. Birth Defects Res A Clin Mol Teratol 2009; 85: 677-694.

2 Pena SD, Shokeir MH: Syndrome of camptodactyly, multiple ankyloses, facial anomalies, and pulmonary hypoplasia: a lethal condition. J Pediatr 1974; 85: 373-375

3 Ravenscroft G, Sollis E, Charles AK, North KN, Baynam G, Laing NG: Fetal akinesia: review of the genetics of the neuromuscular causes. J Med Genet 2011; 48: 793-801.

4 Vogt J, Morgan NV, Rehal P et al: CHRNG genotype-phenotype correlations in the multiple pterygium syndromes. J Med Genet 2012; 49: 21-26.

5 DeChiara TM, Bowen DC, Valenzuela DM et al: The receptor tyrosine kinase MuSK is required for neuromuscular junction formation in vivo. Cell 1996; 85: 501-512.
6 Wierenga KJ, Jiang Z, Yang AC, Mulvihill JJ, Tsinoremas NF: A clinical evaluation tool for SNP arrays, especially for autosomal recessive conditions in offspring of consanguineous parents. Genet Med 2013; 15: 354-360.

7 Meier T, Hauser DM, Chiquet M, Landmann L, Ruegg MA, Brenner HR: Neural agrin induces ectopic postsynaptic specializations in innervated muscle fibers. $J$ Neurosci 1997; 17: 6534-6544

8 Herbst R, Burden SJ: The juxtamembrane region of MuSK has a critical role in agrin-mediated signaling. EMBO J 2000; 19: 67-77.

9 Naldini L, Blömer U, Gallay P et al: In vivo gene delivery and stable transduction of nondividing cells by a lentiviral vector. Science 1996; 272: 263-267.

10 Ferns MJ, Campanelli JT, Hoch W, Scheller RH, Hall Z: The ability of agrin to cluster AChRs depends on alternative splicing and on cell surface proteoglycans. Neuron 1993; 11: 491-502.

11 Glass DJ, Bowen DC, Stitt TN et al: Agrin acts via a MuSK receptor complex. Cell 1996; 85: 513-523.

12 Saldanha J, Singh J, Mahadevan D: Identification of a Frizzled-like cysteine rich domain in the extracellular region of developmental receptor tyrosine kinases. Protein Sci 1998; 7: 1632-1635.

13 Ghazanfari N, Fernandez KJ, Murata $Y$ et al: Muscle specific kinase: organiser of synaptic membrane domains. Int J Biochem Cell Biol 2011; 43: 295-298.

14 Mazhar S, Herbst R: The formation of complex acetylcholine receptor clusters requires MuSK kinase activity and structural information from the MuSK extracellular domain. Mol Cell Neurosci 2012; 49: 475-486.

15 Bergamin E, Hallock PT, Burden SJ, Hubbard SR: The cytoplasmic adaptor protein Dok7 activates the receptor tyrosine kinase MuSK via dimerization. Mol Cell 2010; 39: 100-109.

16 Hopf C, Hoch W: Dimerization of the muscle-specific kinase induces tyrosine phosphorylation of acetylcholine receptors and their aggregation on the surface of myotubes. J Biol Chem 1998; 273: 6467-6473.

17 Fuhrer C, Sugiyama JE, Taylor RG, Hall ZW: Association of muscle-specific kinase MuSK with the acetylcholine receptor in mammalian muscle. EMBO J 1997; 16: 4951-4960.

18 Engel AG: Current status of the congenital myasthenic syndromes. Neuromuscul Disord 2012; 22: 99-111.

19 Michalk A, Stricker S, Becker J et al: Acetylcholine receptor pathway mutations explain various fetal akinesia deformation sequence disorders. Am J Hum Genet 2008; 82: 464-476.

20 Vogt J, Harrison BJ, Spearman $\mathrm{H}$ et al: Mutation analysis of CHRNA1, CHRNB1, CHRND, and RAPSN genes in multiple pterygium syndrome/fetal akinesia patients. Am J Hum Genet 2008; 82: 222-227.

21 Vogt J, Morgan NV, Marton T et al: Germline mutation in DOK7 associated with fetal akinesia deformation sequence. J Med Genet 2009; 46: 338-340.

22 Cossins J, Liu WW, Belaya $\mathrm{K}$ et al: The spectrum of mutations that underlie the neuromuscular junction synaptopathy in DOK7 congenital myasthenic syndrome. Hum Mol Genet 2012; 21: 3765-3775.

23 Chevessier F, Faraut B, Ravel-Chapuis A et al: MUSK, a new target for mutations causing congenital myasthenic syndrome. Hum Mol Genet 2004; 13: 3229-3240.

24 Mihaylova V, Salih MAM, Mukhtar MM et al: Refinement of the clinical phenotype in musk-related congenital myasthenic syndromes. Neurology 2009; 73: 1926-1928.

25 Maselli RA, Arredondo J, Cagney 0 et al: Mutations in MUSK causing congenital myasthenic syndrome impair MuSK-Dok-7 interaction. Hum Mol Genet 2010; 19. 2370-2379.

26 Ben Ammar A, Soltanzadeh P, Bauché S et al: A mutation causes MuSK reduced sensitivity to agrin and congenital myasthenia. PLOS ONE 2013; 8: e53826.

27 Gallenmüller C, Felber WM, Dusl M et al: Salbutamol-responsive limb-girdle congenital myasthenic syndrome due to a novel missense mutation and heteroallelic deletion in MUSK. Neuromuscul Disord 2013; 24: 31-35.

28 Till JH, Becerra M, Watty A et al: Crystal structure of the MuSK tyrosine kinase: insights into receptor autoregulation. Structure 2002; 10: 1187-1196.

Supplementary Information accompanies this paper on European Journal of Human Genetics website (http://www.nature.com/ejhg) 